\title{
Microscopic versus macroscopic approaches to non-equilibrium systems
}

\author{
Bernard Derrida \\ Laboratoire de Physique Statistique, Ecole Normale Supérieure, \\ UPMC Paris 6, Université Paris Diderot Paris 7, CNRS, \\ 24, rue Lhomond, 75231 Paris Cedex 05, France
}

\begin{abstract}
The one dimensional symmetric simple exclusion process (SSEP) is one of the very few exactly soluble models of non-equilibrium statistical physics. It describes a system of particles which diffuse with hard core repulsion on a one dimensional lattice in contact with two reservoirs of particles at unequal densities. The goal of this note is to review the two main approaches which lead to the exact expression of the large deviation functional of the density of the SSEP in its steady state: a microscopic approach (based on the matrix product ansatz and an additivity property) and a macroscopic approach (based on the macroscopic fluctuation theory of Bertini, De Sole, Gabrielli, Jona-Lasinio and Landim).

PACS numbers: 02.50.-r, 05.40.-a, 05.70 Ln, 82-20-w
\end{abstract}

\section{The Symmetric Simple Exclusion Process}

Understanding the steady state properties of systems in contact with two heat baths at unequal temperatures or two reservoirs of particles at unequal densities is a central question in the theory of non-equilibrium systems [1, 2, 3, 4]. Here I would like to focus on one exact result which was obtained during the last decade on the steady state of one of the simplest models of a non-equilibrium system, the one dimensional symmetric simple exclusion process (SSEP). For the SSEP this exact result, which gives an expression of the large deviation functional of the density, can be derived either from a microscopic description of the steady state [5, 6, 7] or from a macroscopic approach, which was developed by Bertini, De Sole, Gabrielli, Jona-Lasinio and Landim [8, 9, 10]. These two approaches are discussed below.

The symmetric simple exclusion process (SSEP) describes a gas of particles diffusing on a lattice with an exclusion rule which prevents a particle to move to a site already occupied by another particle [11, 12, 13, 14, 15]. Here we consider the one dimensional version with open boundaries. The lattice consists of $L$ sites, each site being either occupied by a single particle or empty. During every infinitesimal time interval $d t$, each particle has a probability $d t$ of jumping to the left if the neighboring site on its left is empty, $d t$ of jumping to the right if the neighboring site on its right is empty. At the two 


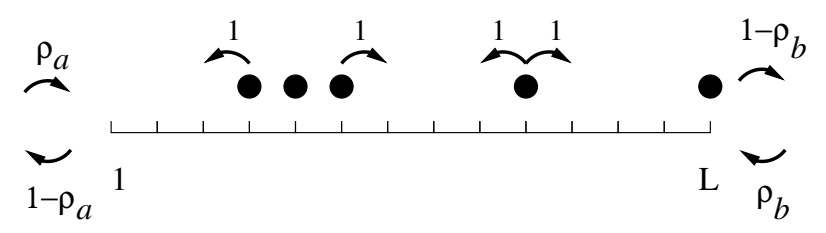

Figure 1. The symmetric simple exclusion process: particles diffuse with hard core repulsion on a one dimensional lattice connected at its ends to two reservoirs of particles at densities $\rho_{a}$ and $\rho_{b}$.

boundaries the dynamics is modified to mimic the coupling with reservoirs of particles at densities $\rho_{a}$ for the left reservoir and $\rho_{b}$ for the right reservoir: at the left boundary, during each time interval $d t$, a particle is injected on site 1 with probability $2 \rho_{a} d t$ (if this site is empty) and a particle is removed from site 1 with probability $2\left(1-\rho_{a}\right) d t$ (if this site is occupied). Similarly on site $L$, particles are injected at rate $2 \rho_{b}$ and removed at rate $2\left(1-\rho_{b}\right)$. (The factors 2 in the boundary rates simplify some expressions below but do not affect the large scale properties).

The SSEP is obviously a model of transport of particles between two reservoirs at densities $\rho_{a}$ and $\rho_{b}$. It is also a simple model of heat transport between two heat baths at temperatures $T_{a}$ and $T_{b}$, if one interprets each particle as a quantum of energy $\epsilon$, with

$$
\exp \left[-\frac{\epsilon}{k T_{a}}\right]=\frac{\rho_{a}}{1-\rho_{a}} \quad ; \quad \exp \left[-\frac{\epsilon}{k T_{b}}\right]=\frac{\rho_{b}}{1-\rho_{b}} .
$$

Under the evolution rules of the SSEP, the system reaches, in the long time limit, a steady state. If one divides the system of length $L$ into $n$ boxes of size $l$ (with of course $L=n l$ ), one can try to determine, in this steady state, the probability of a certain density profile $\left\{r_{1}, r_{2} . . r_{n}\right\}$, i.e. the probability of seeing $l r_{1}$ particles in the first box, $l r_{2}$ particles in the second box, $\ldots l r_{n}$ particles in the $n$th box. For large $L$, one expects the following $L$ dependence of this probability

$$
\operatorname{Pro}_{L}\left(r_{1}, \ldots r_{n} \mid \rho_{a}, \rho_{b}\right) \sim \exp \left[-L \mathcal{F}_{n}\left(r_{1}, r_{2}, \ldots r_{n} \mid \rho_{a}, \rho_{b}\right)\right]
$$

where $\mathcal{F}_{n}\left(r_{1}, r_{2}, \ldots r_{n} \mid \rho_{a}, \rho_{b}\right)$ is called the large deviation function [16] of the density profile $\left\{r_{1}, r_{2} . . r_{n}\right\}$. When the number $n$ of boxes becomes large, keeping the number $l$ of sites in each box also large, one can introduce a continuous variable $x=k / n$, the densities $r_{1}, r_{2} . . r_{n}$ become a density profile

$$
\rho(x)=\rho\left(\frac{k}{n}\right)=r_{k}
$$

and the large deviation function $\mathcal{F}\left(r_{1}, r_{2}, \ldots r_{n} \mid \rho_{a}, \rho_{b}\right)$ becomes a functional $\mathcal{F}\left(\{\rho(x)\} \mid \rho_{a}, \rho_{b}\right)$ of the density profile $\rho(x)$

$$
\operatorname{Pro}_{L}\left(\{\rho(x)\} \mid \rho_{a}, \rho_{b}\right) \sim \exp \left[-L \mathcal{F}\left(\{\rho(x)\} \mid \rho_{a}, \rho_{b}\right)\right] .
$$

The result which was obtained in [5, 9, 6] is that, in the non-equilibrium case, i.e. for $\rho_{a} \neq \rho_{b}$, the exact expression of the large deviation function $\mathcal{F}\left(\{\rho(x)\} \mid \rho_{a}, \rho_{b}\right)$ is given by

$$
\mathcal{F}\left(\{\rho(x)\} \mid \rho_{a}, \rho_{b}\right)=\int_{0}^{1} d x\left[B(\rho(x), F(x))+\log \frac{F^{\prime}(x)}{\rho_{b}-\rho_{a}}\right]
$$


where $B(\rho, r)$ is given by

$$
B(\rho, r)=(1-\rho) \log \frac{1-\rho}{1-r}+\rho \log \frac{\rho}{r}
$$

and where the function $F(x)$ is the monotone solution of the differential equation

$$
\rho(x)=F+\frac{F(1-F) F^{\prime \prime}}{F^{\prime 2}}
$$

satisfying the boundary conditions $F(0)=\rho_{a}$ and $F(1)=\rho_{b}$.

\section{Properties of this large deviation function}

Before describing the two main approaches which led to 456 , let us discuss briefly a few properties of the functional $\mathcal{F}$.

One can solve perturbatively (6) for $\rho_{a}$ close to $\rho_{b}$ and get

$$
\begin{aligned}
F=\rho_{a}-\left(\rho_{a}-\rho_{b}\right) x-\frac{\left(\rho_{a}-\rho_{b}\right)^{2}}{\rho_{a}\left(1-\rho_{a}\right)}[ & (1-x) \int_{0}^{x} y\left(\rho(y)-\rho_{a}\right) d y \\
& \left.+x \int_{x}^{1}(1-y)\left(\rho(y)-\rho_{a}\right) d y\right]+O\left(\left(\rho_{a}-\rho_{b}\right)^{3}\right) .
\end{aligned}
$$

Therefore in the limit $\rho_{b} \rightarrow \rho_{a}$, the expression (4) reduces to

$$
\mathcal{F}\left(\{\rho(x)\} \mid \rho_{a}, \rho_{a}\right)=\int_{0}^{1} d x\left[B\left(\rho(x), \rho_{a}\right)\right]
$$

This is not surprising as, when $\rho_{a}=\rho_{b}$, the dynamics satisfies detailed balance and in the steady state all the lattice sites are occupied independently with probability $\rho_{a}$ (Bernoulli measure). In this equilibrium case $\mathcal{F}$ is a local functional (8) of the density profile $\rho(x)$. This is a special case of the much more general fact [7, 17] that, for any system (with short range interactions) at equilibrium, in contact with one or several reservoirs at density $\rho_{a}$, the functional $\mathcal{F}$ is always local and is given by

$$
\mathcal{F}\left(\{\rho(x)\} \mid \rho_{a}, \rho_{a}\right)=\int_{0}^{1} d x\left[f(\rho(x))-f\left(\rho_{a}\right)-\left(\rho-\rho_{a}\right) f^{\prime}\left(\rho_{a}\right)\right]
$$

where $f(\rho)$ is the free energy per unit volume at density $\rho$, defined as $f(\rho)=$ $-\lim _{V \rightarrow \infty} \log Z(V, V \rho) / V$ where $Z(V, N)$ is the partition function of a system of $N$ particles at equilibrium in a volume $V$.

In the $\rho_{a} \neq \rho_{b}$ case, the large deviation functional $\mathcal{F}$ can therefore be thought as a possible generalization of the concept of free energy to non-equilibrium systems.

As soon as the system is out of equilibrium $\left(\rho_{b} \neq \rho_{a}\right)$ the large deviation functional $\mathcal{F}$ becomes non-local. This is already visible in the expansion of $\mathcal{F}$ in powers of $\rho_{a}-\rho_{b}$, obtained by replacing $F(x)$ by its expansion (7) into (4)

$$
\begin{aligned}
& \mathcal{F}\left(\{\rho(x)\} \mid \rho_{a}, \rho_{b}\right)=\int_{0}^{1} d x B\left(\rho(x), \rho^{*}(x)\right) \\
& +\frac{\left(\rho_{a}-\rho_{b}\right)^{2}}{\left[\rho_{a}\left(1-\rho_{a}\right)\right]^{2}}\left[\int_{0}^{1} d x \int_{x}^{1} d y x(1-y)\left(\rho(x)-\rho^{*}(x)\right)\left(\rho(y)-\rho^{*}(y)\right)\right]+O\left(\rho_{a}-\rho_{b}\right)^{3}
\end{aligned}
$$

where the average profile $\rho^{*}(x)$ is given by

$$
\rho^{*}(x)=(1-x) \rho_{a}+x \rho_{b} .
$$


The knowledge of the functional $\mathcal{F}$ allows one to determine all the the correlation functions: if one defines the generating function $\mathcal{G}$ of the density by

$$
\exp \left[L \mathcal{G}\left(\{\alpha(x)\} \mid \rho_{a}, \rho_{b}\right)\right]=\left\langle\exp \left[L \int_{0}^{1} \alpha(x) \rho(x) d x\right]\right\rangle
$$

where $\alpha(x)$ is an arbitrary function and $\langle$.$\rangle denotes an average over the profile \rho(x)$ in the steady state, it is clear from (3) that, for large $L, \mathcal{G}$ is the Legendre transform of $\mathcal{F}$

$$
\mathcal{G}\left(\{\alpha(x)\} \mid \rho_{a}, \rho_{b}\right)=\max _{\{\rho(x)\}}\left[\int_{0}^{1} \alpha(x) \rho(x) d x-\mathcal{F}\left(\{\rho(x)\} \mid \rho_{a}, \rho_{b}\right)\right] .
$$

By taking derivatives of 12 with respect to $\alpha(x)$ one can then get all the correlation functions. In particular

$$
\langle\rho(x) \rho(y)\rangle_{c} \equiv\langle\rho(x) \rho(y)\rangle-\langle\rho(x)\rangle\langle\rho(y)\rangle=\left.\frac{1}{L} \frac{\delta^{2} \mathcal{G}}{\delta \alpha(x) \delta \alpha(y)}\right|_{\alpha(x)=0}
$$

A direct consequence of 12 is that all the $k$-point connected correlation functions are long ranged and scale like $L^{1-k}$ (see [18, 19, 7]).

\section{The steady state}

From the definition of the SSEP, if $\tau_{i}$ is a binary variable with $\tau_{i}=1$ when site $i$ is occupied and $\tau_{i}=0$ when it is empty, one can write the time evolution of the average occupation $\left\langle\tau_{i}\right\rangle$

$$
\begin{aligned}
& \frac{d\left\langle\tau_{1}\right\rangle}{d t}=2 \rho_{a}-3\left\langle\tau_{1}\right\rangle+\left\langle\tau_{2}\right\rangle \\
& \frac{d\left\langle\tau_{i}\right\rangle}{d t}=\left\langle\tau_{i-1}\right\rangle-2\left\langle\tau_{i}\right\rangle+\left\langle\tau_{i+1}\right\rangle \quad \text { for } \quad 2 \leq i \leq L-1 \\
& \frac{d\left\langle\tau_{L}\right\rangle}{d t}=\left\langle\tau_{L-1}\right\rangle-3\left\langle\tau_{L}\right\rangle+2 \rho_{b} .
\end{aligned}
$$

The steady state density profile (obtained by writing that $\frac{d\left\langle\tau_{i}\right\rangle}{d t}=0$ ) is [6]

$$
\left\langle\tau_{i}\right\rangle=\frac{(L+1-2 i) \rho_{a}+(2 i-1) \rho_{b}}{2 L}
$$

For large $L$, with $i=L x$, one recovers the average density profile (11).

In a similar way one can then write down the equations which govern the time evolution of the two point function or higher correlations.

\section{The matrix ansatz for the SSEP}

For the SSEP, one can then write down the steady state equations satisfied by higher and higher correlation functions, but solving these equations becomes quickly complicated.

The matrix ansatz [20, 21, 22] gives an algebraic way of calculating exactly the weights of all the configurations in the steady state: in [20] it was shown that, in the 
steady state, the probability of a microscopic configuration $\left\{\tau_{1}, \tau_{2}, \ldots \tau_{L}\right\}$ can be written as the matrix element of a product of $L$ matrices

$$
\operatorname{Pro}\left(\left\{\tau_{1}, \tau_{2}, \ldots \tau_{L}\right\}\right)=\frac{\left\langle\rho_{a}\left|X_{1} X_{2} \ldots X_{L}\right| \rho_{b}\right\rangle}{\left\langle\rho_{a}\left|(D+E)^{L}\right| \rho_{b}\right\rangle}
$$

where the matrix $X_{i}$ depends on the occupation number $\tau_{i}$

$$
X_{i}=\tau_{i} D+\left(1-\tau_{i}\right) E,
$$

and the matrices $D, E$ and the vectors $\left\langle\rho_{a}|,| \rho_{b}\right\rangle$ satisfy the following algebraic rules

$$
\begin{aligned}
& D E-E D=D+E \\
& \left\langle\rho_{a}\right| 2\left[\rho_{a} E-\left(1-\rho_{a}\right) D\right]=\left\langle\rho_{a}\right| \\
& 2\left[\left(1-\rho_{b}\right) D-\rho_{b} E\right]\left|\rho_{b}\right\rangle=\left|\rho_{b}\right\rangle .
\end{aligned}
$$

A priori one should construct the matrices $D$ and $E$ (which might be infinitedimensional) and the vectors $\left\langle\rho_{a}\right|$ and $\left|\rho_{b}\right\rangle$ satisfying (19) to calculate the weights of the microscopic configurations. However these weights do not depend on the particular representation chosen and can be calculated directly [20, 7] from (19).

One can calculate, using (19), the average density profile

$$
\left\langle\tau_{i}\right\rangle=\frac{\left\langle\rho_{a}\left|(D+E)^{i-1} D(D+E)^{L-i}\right| \rho_{b}\right\rangle}{\left\langle\rho_{a}\left|(D+E)^{L}\right| \rho_{b}\right\rangle}
$$

as well as all the correlation functions and recover $(16)$. One can also show that

$$
\frac{\left\langle\rho_{a}\left|(D+E)^{L}\right| \rho_{b}\right\rangle}{\left\langle\rho_{a} \mid \rho_{b}\right\rangle}=\frac{L !}{\left(\rho_{a}-\rho_{b}\right)^{L}} .
$$

(This formula is easy to derive by noticing that, for a system of size $L$, the average current is given, according to (16), by $\left\langle\tau_{i}-\tau_{i+1}\right\rangle=\left(\rho_{a}-\rho_{b}\right) / L$ but is also given, according to 2019), by the ratio $\left.\left\langle\rho_{a}\left|(D+E)^{L-1}\right| \rho_{b}\right\rangle /\left\langle\rho_{a}\left|(D+E)^{L}\right| \rho_{b}\right\rangle\right)$.

\section{Additivity}

As in (17) the weight of each configuration is written as the matrix element of a product of $L$ matrices, one can try to insert at a position $L_{1}$ a complete basis in order to relate the properties of a lattice of $L$ sites to those of two subsystems of sizes $L_{1}$ and $L-L_{1}$.

If one defines, for arbitrary $\rho$, left and right vectors $\langle\rho|$ and $|\rho\rangle$, which satisfy

$$
\begin{aligned}
& \langle\rho| 2[\rho E-(1-\rho) D]=\langle\rho| \\
& 2[(1-\rho) D-\rho E]|\rho\rangle=|\rho\rangle
\end{aligned}
$$

(note that in general $\left\langle\rho \mid \rho^{\prime}\right\rangle \neq 0$ ), it is possible to show, using $D E-E D=D+E$ as in (19) and the property (22), that for $\rho_{b}<\rho_{a}$

$$
\frac{\left\langle\rho_{a}\left|Y_{1} Y_{2}\right| \rho_{b}\right\rangle}{\left\langle\rho_{a} \mid \rho_{b}\right\rangle}=\oint_{\rho_{b}<|\rho|<\rho_{a}} \frac{d \rho}{2 i \pi} \frac{\left(\rho_{a}-\rho_{b}\right)}{\left(\rho_{a}-\rho\right)\left(\rho-\rho_{b}\right)} \frac{\left\langle\rho_{a}\left|Y_{1}\right| \rho\right\rangle}{\left\langle\rho_{a} \mid \rho\right\rangle} \frac{\left\langle\rho\left|Y_{2}\right| \rho_{b}\right\rangle}{\left\langle\rho \mid \rho_{b}\right\rangle}
$$

where $Y_{1}$ and $Y_{2}$ are arbitrary polynomials of matrices $D$ and $E$. (To prove (23), one can first prove it, using (21), for $Y_{1}$ of the form $\left[\rho_{a} E-\left(1-\rho_{a}\right) D\right]^{m_{1}}[D+E]^{n_{1}}$ and $Y_{2}$ of 
the form $[D+E]^{n_{2}}\left[\left(1-\rho_{b}\right) D-\rho_{b} E\right]^{m_{2}}$. Then one can show, using $D E-E D=D+E$, that any polynomial $Y_{1}$ or $Y_{2}$ can be reduced to a finite sum of such terms).

By choosing for $Y_{1}$ the sum over the weights of all configurations with $l r_{1}$ occupied sites in the first a box, $\ldots l r_{k}$ occupied sites in the $k$ th box, and for $Y_{2}$ the sum over all configurations with $l r_{k+1}$ occupied sites in the $k+1$ th box, $\ldots l r_{n}$ occupied sites in the $n$th box, one can show, using $(17|21| 23)$ that

$$
\begin{array}{r}
\operatorname{Pro}_{L}\left(r_{1}, \ldots r_{n} \mid \rho_{a}, \rho_{b}\right)=\oint_{\rho_{b}<|\rho|<\rho_{a}} \frac{d \rho}{2 i \pi} \frac{L_{1} !\left(L-L_{1}\right) !}{L !} \frac{\left(\rho_{a}-\rho_{b}\right)^{L+1}}{\left(\rho_{a}-\rho\right)^{L_{1}+1}\left(\rho-\rho_{b}\right)^{L-L_{1}+1}} \times \\
\operatorname{Pro}_{L_{1}}\left(r_{1}, \ldots r_{k} \mid \rho_{a}, \rho\right) \times \operatorname{Pro}_{L-L_{1}}\left(r_{k+1}, \ldots r_{n} \mid \rho, \rho_{b}\right)
\end{array}
$$

where $L_{1}=k l$. This formula, which is exact for arbitrary system sizes, relates the properties of two disconnected subsystems of sizes $L_{1}$ and $L-L_{1}$ to those of a single system of size $L$.

If $L_{1}=L x$, one then gets (2) for large $L$

$$
\begin{aligned}
\mathcal{F}_{n}\left(r_{1}, r_{2}, \ldots r_{n} \mid \rho_{a}, \rho_{b}\right)= & \max _{\rho_{b}<F<\rho_{a}}\left[x \mathcal{F}_{k}\left(r_{1}, \ldots r_{k} \mid \rho_{a}, F\right)+(1-x) \mathcal{F}_{n-k}\left(r_{k+1}, \ldots r_{n} \mid F, \rho_{b}\right)\right. \\
& \left.+x \log \left(\frac{\rho_{a}-F}{x}\right)+(1-x) \log \left(\frac{F-\rho_{b}}{1-x}\right)-\log \left(\rho_{a}-\rho_{b}\right)\right]
\end{aligned}
$$

which follows from (24) by a saddle point method (as in 24) the integration contour is perpendicular to the real axis, the value $F$ of $\rho$ which maximizes the integrand along the contour becomes a minimum as $\rho$ varies along the real axis). If one repeats the same procedure $n$ times, one gets

$\mathcal{F}_{n}\left(r_{1}, r_{2}, \ldots r_{n} \mid \rho_{a}, \rho_{b}\right)=\max _{\rho_{a}=F_{0}>. .>F_{k}>. .>F_{n}=\rho_{b}} \frac{1}{n} \sum_{k=1}^{n} \mathcal{F}_{1}\left(r_{k} \mid F_{k-1}, F_{k}\right)+\log \left(\frac{\left(F_{k-1}-F_{k}\right) n}{\rho_{a}-\rho_{b}}\right)$

For large $n$, as $F_{k}$ is monotone, the difference $F_{k-1}-F_{k}$ has to be small for almost all $k$ and one can replace $\mathcal{F}_{1}\left(r_{k} \mid F_{k-1}, F_{k}\right)$ by its equilibrium value $\mathcal{F}_{1}\left(r_{k} \mid F_{k}, F_{k}\right)=B\left(r_{k}, F_{k}\right)$ (see (8)). If one the writes $F_{k}$ as a function of $k / n$

$$
F_{k}=F\left(\frac{k}{n}\right)
$$

(26) becomes

$$
\mathcal{F}\left(\{\rho(x)\} \mid \rho_{a}, \rho_{b}\right)=\max _{F(x)} \int_{0}^{1} d x\left[B(\rho(x), F(x))+\log \frac{F^{\prime}(x)}{\rho_{b}-\rho_{a}}\right]
$$

where the maximun is over all the monotone functions $F(x)$ which satisfy $F(0)=\rho_{a}$ and $F(1)=\rho_{b}$. Writing the equation satified by the optimal $F(x)$ in (28) leads to (6) and this completes the derivation of $(4,6)$. 


\section{The macroscopic fluctuation theory}

For a general diffusive one dimensional system of length $L$, in contact with a left reservoir at density $\rho_{a}$ and a right reservoir at density $\rho_{b}$, the average current and the fluctuations of this current near equilibrium can be characterized by two quantities $D(\rho)$ and $\sigma(\rho)$ defined by

$$
\begin{aligned}
& \lim _{t \rightarrow \infty} \frac{\left\langle Q_{t}\right\rangle}{t}=\frac{D(\rho)}{L}\left(\rho_{a}-\rho_{b}\right) \quad \text { for }\left(\rho_{a}-\rho_{b}\right) \text { small } \\
& \lim _{t \rightarrow \infty} \frac{\left\langle Q_{t}^{2}\right\rangle}{t}=\frac{\sigma(\rho)}{L} \quad \text { for } \rho_{a}=\rho_{b}
\end{aligned}
$$

where $Q_{t}$ is the total number of particles transferred from the left reservoir to the system during time $t$. Starting from the hydrodynamic large deviation theory [23, 18, 14] Bertini, De Sole, Gabrielli, Jona-Lasinio and Landim [8, 9, 10] have developed a general approach, the macroscopic fluctuation theory, to calculate the large deviation functional $\mathcal{F}$ of the density (3) in the non-equilibrium steady state of a diffusive system in contact with two reservoirs as in figure 1 .

For diffusive systems (such as the SSEP), the density $\rho_{i}(t)$ near site $i$ at time $t$ and the total flux $Q_{i}(t)$ flowing through position $i$ between time 0 and time $t$ are, for a large system of size $L$ and for times of order $L^{2}$, scaling functions of the form

$$
\rho_{i}(t)=\widehat{\rho}\left(\frac{i}{L}, \frac{t}{L^{2}}\right), \quad \text { and } \quad Q_{i}(t)=L \widehat{Q}\left(\frac{i}{L}, \frac{t}{L^{2}}\right)
$$

(Note that, due to the conservation of the number of particles $\rho_{i}(t)-\rho_{i}(0)=Q_{i}(t)-$ $Q_{i+1}(t)$, the scaling form of $\rho_{i}(t)$ implies the scaling form of $\left.Q_{i}(t)\right)$. If one introduces the instantaneous (rescaled) current defined by

$$
\widehat{j}(x, \tau)=\frac{\partial \widehat{Q}(x, \tau)}{\partial \tau}
$$

the conservation of the number of particles implies that

$$
\frac{\partial \widehat{\rho}(x, \tau)}{\partial \tau}=-\frac{\partial^{2} \widehat{Q}(x, \tau)}{\partial \tau \partial x}=-\frac{\partial \widehat{j}(x, \tau)}{\partial x} .
$$

The macroscopic fluctuation theory [8, 9, 10] starts from the probability of observing a certain density profile $\widehat{\rho}(x, \tau)$ and current profile $\widehat{j}(x, \tau)$ over the rescaled time interval $\tau_{1}<\tau<\tau_{2}$

$\mathcal{P}_{\tau_{1}, \tau_{2}}(\{\widehat{\rho}(x, \tau), \widehat{j}(x, \tau)\}) \sim \exp \left[-L \int_{\tau_{1}}^{\tau_{2}} d \tau^{\prime} \int_{0}^{1} d x \frac{\left[\widehat{j}\left(x, \tau^{\prime}\right)+D\left(\widehat{\rho}\left(x, \tau^{\prime}\right)\right) \frac{\partial \widehat{\rho}\left(x, \tau^{\prime}\right)}{\partial x}\right]^{2}}{2 \sigma\left(\widehat{\rho}\left(x, \tau^{\prime}\right)\right)}\right]$

where the current $\hat{j}(x, s)$ is related to the density profile $\widehat{\rho}(x, s)$ by the conservation law (33) and the functions $D(\rho)$ and $\sigma(\rho)$ are defined by 2930$)$. The physical meaning of (34) is that the system is locally close to equilibrium and that the fluctuations of the local currents are Gaussian with averages and variances given by (29) 30$)$.

Then to calculate the probability of observing a density profile $\rho(x)$ in the steady state, at time $\tau$, one has to find how this profile is produced. For large $L$, this probability 
(34) is dominated by the optimal path $\{\widehat{\rho}(x, s), \widehat{j}(x, s)\}$ for $-\infty<s<\tau$ in the space of density and current profiles and

$$
\operatorname{Pro}_{L}\left(\{\rho(x)\} \mid \rho_{a}, \rho_{b}\right) \sim \max _{\{\widehat{\rho}(x, s), \widehat{j}(x, s)\}} \mathcal{P}_{-\infty, \tau}(\{\widehat{\rho}(x, s), \hat{j}(x, s)\})
$$

which goes from the average steady state profile $\rho^{*}(x)$ (given by (11) for the SSEP) to the desired profile $\rho(x)$

$$
\widehat{\rho}(x,-\infty)=\rho^{*}(x) \quad ; \quad \widehat{\rho}(x, \tau)=\rho(x) .
$$

This means that the functional $\mathcal{F}$ of the density $(3)$ is given by

$\mathcal{F}\left(\{\rho(x)\} \mid \rho_{a}, \rho_{b}\right)=\min _{\{\widehat{\rho}(x, s), \widehat{j}(x, s)\}} \int_{-\infty}^{\tau} d \tau^{\prime} \int_{0}^{1} d x \frac{\left[\widehat{j}\left(x, \tau^{\prime}\right)+D\left(\widehat{\rho}\left(x, \tau^{\prime}\right)\right) \frac{\partial \widehat{\rho}\left(x, \tau^{\prime}\right)}{\partial x}\right]^{2}}{2 \sigma\left(\widehat{\rho}\left(x, \tau^{\prime}\right)\right)}$

where the density and the current profiles satisfy the conservation law (33) and the boundary conditions 36 .

Finding this optimal path $\hat{\rho}(x, s), \hat{j}(x, s)$ with the boundary conditions 36 is usually a hard problem. Bertini et al [8] were however able to write an equation satisfied by $\mathcal{F}$ : as 37 does not depend on $\tau$ (because the probability of producing a certain deviation $\rho(x)$ in the steady state does not depend on the time $\tau$ at which this deviation occurs), one can isolate in the integral (37) the contribution of the last time interval $(\tau-\delta \tau, \tau)$ and $(37)$ becomes

$\mathcal{F}(\{\rho(x)\})=\min _{\delta \rho(x), j(x)}\left[\mathcal{F}(\{\rho(x)-\delta \rho(x)\})+\delta \tau \int_{0}^{1} d x \frac{\left[j(x)+D(\rho(x)) \rho^{\prime}(x)\right]^{2}}{2 \sigma(\rho(x))}\right]$

where $\rho(x)-\delta \rho(x)=\widehat{\rho}(x, \tau-d \tau)$ and $j(x)=\widehat{j}(x, \tau)$. Then if one defines $U(x)$ by

$$
U(x)=\frac{\delta \mathcal{F}(\{\rho(x)\})}{\delta \rho(x)}
$$

and one uses the conservation law $\delta \rho(x)=-\frac{d j(x)}{d x} d \tau$, one should have according to 38 that the optimal current $j(x)$ is given by

$$
j(x)=-D(\rho(x)) \rho^{\prime}(x)+\sigma(\rho(x)) U^{\prime}(x) .
$$

Therefore "starting" with $\hat{\rho}(x, \tau)=\rho(x)$ at time $\tau$ and using the time evolution (for $-\infty<s<\tau)$

$$
\frac{d \widehat{\rho}(x, s)}{d s}=-\frac{d \widehat{j}(x, s)}{d x}
$$

with $\widehat{j}$ related to $\hat{\rho}$ as in 40 one should get the whole time dependent optimal profile $\widehat{\rho}(x, s)$ which converges to $\rho^{*}(x)$ in the limit $s \rightarrow-\infty$. The problem of course is that $\mathcal{F}$ is in general not known and so is $U(x)$ defined in (39).

One can write from (38) (after an integration by parts and using the fact that $U(0)=U(1)=0$ if $\rho(0)=\rho_{a}$ and $\left.\rho(1)=\rho_{b}\right)$ the equation satisfied by $U^{\prime}(x)$

$$
\int_{0}^{1} d x\left[\left(\frac{D \rho^{\prime}}{\sigma}-U^{\prime}\right)^{2}-\left(\frac{D \rho^{\prime}}{\sigma}\right)^{2}\right] \frac{\sigma}{2}=0
$$


which is the Hamilton-Jacobi equation of Bertini et al [8]. For general $D(\rho)$ and $\sigma(\rho)$ one does not know how to find the solution $U^{\prime}(x)$ of 42 for an arbitrary $\rho(x)$ and thus one does not know how to get a more explicit expression of the large deviation function $\mathcal{F}(\{\rho(x)\})$.

One can however check rather easily whether a given expression of $\mathcal{F}$ satisfies (42) since $U^{\prime}(x)$ can be calculated from (39). For the SSEP one gets from 28 39)

$$
U(x)=\log \left[\frac{\rho(x)(1-F(x))}{(1-\rho(x)) F(x)}\right]
$$

with $F(x)$ related to $\rho(x)$ by (6). One can then check that (42) is indeed satisfied using the known expressions of $D=1$ and $\sigma=2 \rho(1-\rho)$ for the SSEP [7] (using the fact that $F^{\prime \prime}(0)=F^{\prime \prime}(1)=0$ which is a consequence of the fact that $\rho(0)=F(0)=\rho_{a}$, $\rho(1)=F(1)=\rho_{b}$, and of (6) $)$.

In fact when $\mathcal{F}$ is known, one can obtain the whole optimal path $\widehat{\rho}(x, s)$ from the evolution 411) with $\hat{j}$ related to $\widehat{\rho}$ by 40 which becomes for the SSEP

$$
\widehat{j}(x, s)=-\frac{d \widehat{\rho}(x, s)}{d x}+\sigma(\widehat{\rho}(x, s)) \log \left[\frac{\widehat{\rho}(x, s)(1-\widehat{F}(x, s))}{(1-\widehat{\rho}(x, s)) \hat{F}(x, s)}\right]
$$

where $\widehat{F}$ is related to $\widehat{\rho}$ by (6). For (4) to coincide with (37), the optimal profile $\widehat{\rho}$ evolving according to (41) should converge to $\rho^{*}(x)$ as $s \rightarrow-\infty$. One can check that this evolution (41) of $\hat{\rho}(x, s)$ for this current (44) is equivalent to the following evolution [9] of $\widehat{F}$

$$
\frac{d \widehat{F}(x, s)}{d s}=-\frac{d^{2} \widehat{F}(x, s)}{d x^{2}}
$$

where $\widehat{F}$ is related to $\widehat{\rho}$ by (6). Clearly (45) is a diffusion equation. Because $F(0)=\rho_{a}$, $F(1)=\rho_{b}$ and because of the minus sign in (45), $\widehat{F}(x, s) \rightarrow \rho^{*}(x)$ as $s \rightarrow-\infty$. Therefore, due to (6), the density $\hat{\rho}(x, s) \rightarrow \rho^{*}(x)$ as $s \rightarrow-\infty$. Thus (41 44) do give the optimal path in (37) with the right boundary conditions (36) and (37) coincides for the SSEP with the prediction (446) of the matrix approach. From (45) 6 ) one can show that the time evolution of a deviation $\hat{\rho}(x, s)$, when it is produced is given, for small $\rho_{a}-\rho_{b}$, by $\frac{d \hat{\rho}(x, s)}{d s}=\frac{d^{2} \widehat{\rho}(x, s)}{d x^{2}}-2 \frac{\left(\rho_{a}-\rho_{b}\right)}{\rho_{a}\left(1-\rho_{a}\right)}(1-2 \widehat{\rho}(x, s)) \frac{d \widehat{\rho}(x, s)}{d s}+O\left(\left(\rho_{a}-\rho_{b}\right)^{2}\right)$.

One can notice that as soon as $\rho_{a} \neq \rho_{b}$ this is not the time reversal of the way a deviation relaxes (15)

$$
\frac{d \rho(x, t)}{d t}=\frac{d^{2} \rho(x, t)}{d x^{2}}
$$

This again is not a surprise as for non-equilibrium systems $\left(\rho_{a} \neq \rho_{b}\right)$, the way a deviation is produced 46 has no reason to be the time reversal of the way it relaxes (47). 


\section{Conclusion}

In addition to the two approaches discussed above to obtain (4-6), Tailleur Kurchan and Lecomte [24] have developed a third approach based on a non-local change of variables which allows them to map the dynamics of the non-equilibrium case $\left(\rho_{a} \neq \rho_{b}\right)$ onto the dynamics of the equilibrium case $\left(\rho_{a}=\rho_{b}\right)$.

Apart from the SSEP (and zero range processes for which the steady state measure is a product measure), the large deviation function $\mathcal{F}$ has been determined so far only for few other cases: the Kipnis Marchioro Presutti model [27, 28], the weakly asymmetric exlcusion process [25, 26], the ABC model [29, 30] on a ring for equal densities of the three species, driven systems [31] in particular the asymmetric exclusion process [32, 33].

An open question is whether one could use the macroscopic fluctuation theory to find the large deviation functional $\mathcal{F}$ for more general diffusive systems characterized by arbitrary functions $D(\rho)$ and $\sigma(\rho)$ defined in 2930 .

More recently, the macroscopic fluctuation theory has become a very powerful tool to calculate the large deviation function of the current in the non-equilibrium steady state of diffusive systems [34, 35, 36, 37, 38, 39, 40, 41, 42]. On the other hand exact calculations of the current fluctuations, starting from a microscopic model, are still very difficult to do [43, 44, 45, 46]. What the large deviation functional of the density looks like, for a diffusive system, when conditioned on the current, remains an open question [47.

Looking, by a macroscopic or a microscopic approach, at diffusive systems with an initial condition which is not a steady state as in [48, 49], would be another interesting direction to pursue.

Lastly, one knows [2, 50] that mechanical systems which conserve momentum exhibit an anomalous Fourier's law in one dimension. What the large deviation functions

of the current or of the density become for such systems looks to me another interesting and challenging question.

\section{Acknowledgments}

My interest for the large deviations of the density of diffusive systems started by the works done with Joel Lebowitz and Gene Speer in 2001-2003. We used, as a starting point, the matrix ansatz which was developed in collaboration with Martin Evans,Vincent Hakim, Vincent Pasquier, following an earlier paper with David Mukamel and Eytan Domany at the beginning of the 1990's. They were pursued by a series of works on diffusive systems, in particular with Thierry Bodineau, Camille Enaud and Antoine Gerschenfeld. It was for me a great pleasure and privilege to work on these problems with them. As the talk, on which this paper is based, was delivered on the occasion of the Boltzmann Medal award, I would like to thank also all my other collaborators or colleagues with whom I had the opportunity to share my interest for Statistical Physics over the past 35 years. 


\section{References}

[1] Bonetto F, Lebowitz J L, Rey-Bellet L Fourier's law: a challenge to theorists 2000 Imperial College Press 128-150 (Preprint math-ph/0002052)

[2] Lepri S, Livi R, Politi A Thermal conduction in classical low-dimensional lattices 2003 Phys. Rep. 377 1-80

[3] Eckmann J P, Pillet C A, Rey-Bellet L Entropy production in nonlinear, thermally driven Hamiltonian systems 1999 J. Stat. Phys. 95 305-331

[4] Sasa S I, Tasaki H Steady state thermodynamics 2006 J. Stat. Phys. 125 125-227

[5] Derrida B, Lebowitz J L, Speer E R Free energy functional for nonequilibrium systems: an exactly solvable case 2001 Phys. Rev. Lett. 87150601

[6] Derrida B, Lebowitz J L, Speer E R Large deviation of the density profile in the steady state of the open symmetric simple exclusion process 2002 J. Stat. Phys. 107 599-634

[7] Derrida B Non-equilibrium steady states: fluctuations and large deviations of the density and of the current 2007 J. Stat. Mech. P07023

[8] Bertini L, De Sole A, Gabrielli D, Jona-Lasinio G, Landim C Fluctuations in stationary non equilibrium states of irreversible processes 2001 Phys. Rev. Lett. 87040601

[9] Bertini L, De Sole A, Gabrielli D, Jona-Lasinio G, Landim C Macroscopic fluctuation theory for stationary non equilibrium states 2002 J. Stat, Phys. 107 635-675

[10] Bertini L, De Sole A, Gabrielli D, Jona-Lasinio G, Landim C Large deviation approach to non equilibrium processes in stochastic lattice gases 2006 Bull. Braz. Math. Soc. 37 611-643

[11] Richards P M Theory of one-dimensional hopping conductivity and diffusion 1977 Phys. Rev. B 16 1393-1409

[12] Spohn H 1991, Large scale dynamics of interacting particles (Springer-Verlag, Berlin)

[13] Liggett T 1999 Stochastic interacting systems: contact, voter and exclusion processes, 324 (Springer-Verlag, Berlin)

[14] Kipnis C, Landim C 1999 Scaling limits of interacting particle systems Springer

[15] Santos J E, Schütz G M Exact time-dependent correlation functions for the symmetric exclusion process with open boundary 2001 Phys. Rev. E 64036107

[16] Touchette H 2009 The large deviation approach to statistical mechanics Phys. Rep. 478 1-69

[17] Bertini L, De Sole A, Gabrielli D, Jona-Lasinio G, Landim C Towards a nonequilibrium thermodynamics: a self-contained macroscopic description of driven diffusive systems $2009 \mathrm{~J}$. Stat. Phys. 135, 857-872

[18] Spohn H Long range correlations for stochastic lattice gases in a non-equilibrium steady state 1983 J. Phys. A 16 4275-4291

[19] Derrida B, Lebowitz J L, Speer E R Entropy of open lattice systems 2007 J. Stat. Phys. 126 1083-1108

[20] Derrida B, Evans M R, Hakim V, Pasquier V Exact solution of a 1d asymmetric exclusion model using a matrix formulation 1993 J. Phys. A 26 1493-1517

[21] Derrida B An exactly soluble non-equilibrium system: the asymmetric exclusion process 1998 Phys. Rep. $301 \quad 65-83$

[22] Blythe R A, Evans M R Nonequilibrium steady states of matrix-product form: a solver's guide 2007 J. Phys. A 40 R333-R441

[23] Kipnis C, Olla S, Varadhan S R S, Hydrodynamics and large deviations for simple exclusion processes 1989 Commun. Pure Appl. Math. 42 115-137

[24] Tailleur J, Kurchan J, Lecomte V Mapping out-of-equilibrium into equilibrium in one-dimensional transport models 2008 J. Phys. A 41505001

[25] Enaud C, Derrida B Large deviation functional of the weakly asymmetric exclusion process 2004 J. Stat. Phys. 114 537-562

[26] Bertini L, Landim C, Mourragui M Dynamical large deviations for the boundary driven weakly asymmetric exclusion process 2009 Ann. Prob. 37 2357-2403 
[27] Kipnis C, Marchioro C, Presutti E, Heat-flow in an exactly solvable model 1982 J. Stat. Phys. 27 65-74

[28] Bertini L , Gabrielli D, Lebowitz J L Large deviation for a stochastic model of heat flow $2005 \mathrm{~J}$. Stat. Phys. 121 843-885

[29] Evans M R, Kafri Y, Koduvely H M, Mukamel D Phase separation in one-dimensional driven diffusive systems 1998 Phys. Rev. Lett. 80 425-429

[30] Clincy M, Derrida B, Evans M R Phase transitions in the ABC model 2003 Phys. Rev. E 67066115

[31] Bahadoran C 2010 A quasi-potential for conservation laws with boundary conditions Preprint math-ph/1010.3624

[32] Derrida B, Lebowitz J L, Speer E R Exact free energy functional for a driven diffusive open stationary nonequilibrium system 2002 Phys. Rev. Lett. 89030601

[33] Derrida B, Lebowitz J L, Speer E R Exact large deviation functional of a stationary open driven diffusive system: the asymmetric exclusion process 2003 J. Stat. Phys. 110 775-810

[34] Bodineau T, Derrida B Current fluctuations in nonequilibrium diffusive systems: an additivity principle 2004 Phys. Rev. Lett. 92180601

[35] Bodineau T, Derrida B Distribution of current in nonequilibrium diffusive systems and phase transitions 2005 Phys. Rev. E 72066110

[36] Bertini L, De Sole A, Gabrielli D, Jona-Lasinio G, Landim C Current fluctuations in stochastic lattice gases 2005 Phys. Rev. Lett. 94030601

[37] Bertini L, De Sole A, Gabrielli D, Jona-Lasinio G, Landim C Non equilibrium current fluctuations in stochastic lattice gases 2006 J. Stat. Phys. 123 237-276

[38] Harris R J, Schütz GM Fluctuation theorems for stochastic dynamics 2007 J. Stat. Mech. P07020

[39] Appert C, Derrida B, Lecomte V, Van Wijland F Universal cumulants of the current in diffusive systems on a ring 2008 Phys. Rev. E $\mathbf{7 8} 021122$

[40] Hurtado P I, Garrido P L Test of the additivity principle for current fluctuations in a model of heat conduction 2009 Phys. Rev. Lett. 102250601

[41] Hurtado P I, Garrido P L Current fluctuations and statistics during a large deviation event in an exactly solvable transport model 2009 J. Stat. Mech: Theory Exp. P02032

[42] Imparato A, Lecomte V, van Wijland F Equilibriumlike fluctuations in some boundary-driven open diffusive systems 2009 Phys. Rev. E 80011131

[43] de Gier J, Essler F H Bethe ansatz solution of the asymmetric exclusion process with open boundaries 2005 Phys. Rev. Lett. 95240601

[44] de Gier J, Essler F H Slowest relaxation mode of the partially asymmetric exclusion process with open boundaries 2008 J. Phys. A 41485002

[45] Prolhac S, Mallick K Cumulants of the current in a weakly asymmetric exclusion process $2009 \mathrm{~J}$. Phys. A 42175001

[46] Simon D Construction of a coordinate Bethe Ansatz for the asymmetric exclusion process with open boundaries 2009 J. Stat. Mech. P07017

[47] Popkov V, Simon D, Schütz G M Asymmetric simple exclusion process on a ring conditioned on enhanced flux 2010 J. Stat. Mech. P07017

[48] Derrida B, Gerschenfeld A Current fluctuations of the one dimensional symmetric simple exclusion process with step initial Condition 2009 J. Stat. Phys. 136 1-15

[49] Derrida B, Gerschenfeld A Current fluctuations in one dimensional diffusive systems with a step initial density profile 2009 J. Stat. Phys. 137 978-1000

[50] Basile G, Bernardin C, Olla S Momentum conserving model with anomalous thermal conductivity in low dimensional systems 2006 Phys. Rev. Lett. 96204303 